\title{
ON THE CAUCHY PROBLEM FOR THE NONLINEAR KLEIN-GORDON EQUATION WITH A CUBIC CONVOLUTION
}

\author{
By
}

\section{Takahiro MotAI}

\begin{abstract}
We study the Cauchy problem for the nonlinear KleinGordon equation with a cubic convolution $\left\{V_{r} *(w(t))^{2}\right\} w(t)$, where $V_{\gamma}(x)=|x|^{-\gamma}$, in $(x, t) \in \boldsymbol{R}^{n} \times \boldsymbol{R}$. We prove the existence of weak solutions for $0<\gamma<n$. We also prove that for $0<\gamma<\operatorname{Min}\{4, n\}$ the weak solution is unique and there exists a regular solution.'
\end{abstract}

Key Words. nonlinear Klein-Gordon equation, cubic convolution, Cauchy problem, global solution, uniqueness.

\section{Introduction and Results.}

We consider the Cauchy problem for the nonlinear Klein-Gordon equation;

$$
\left\{\begin{array}{l}
\partial_{t}^{2} w(t)-\Delta w(t)+w(t)+F(w(t))=0 \\
w(0)=\phi(x), \quad \partial_{t} w(0)=\phi(x)
\end{array}\right.
$$

in $(x, t) \in \boldsymbol{R}^{n} \times \boldsymbol{R}$. Here $w(t)$ is a real valued function and

$$
F(w(t))=\left\{V_{r} * f(w(t))\right\} w(t),
$$

where $f(w)=w^{2}, V_{\gamma}(x)=|x|^{-\gamma}(0<\gamma<n)$ and $*$ denotes the spatial convolution. The study of this equation was begun in Strauss [13] and Menzala and Strauss [9]. In [9] they proved the existence of a global regular solution of (1.1) for $0<\gamma \leqq 3$. The main purpose of the present paper is to prove the same result for $0<\gamma<\operatorname{Min}\{4, n\}$. The upper bound $\operatorname{Min}\{4, n\}$ of $\gamma$ has been already appeared in the case of nonlinear Schrödinger equation with the same nonlinear term. The case of Schrödinger equation has been studied by Chadam and Glassey [2], Glassey [6], Ginibre and Velo [4] and Hayashi and Tsutsumi [7]. It seems that $\operatorname{Min}\{4, n\}$ is a critical value caused by the Sobolev embedding theorem.

In order to state our results, we give the main notations used in this paper. We denote by $\|\cdot\|_{p}$ the norm in $L_{p}=L_{p}\left(\boldsymbol{R}^{n}\right)$. Let $H_{p}^{s}=H_{p}^{s}\left(\boldsymbol{R}^{n}\right)$ with $s \in \boldsymbol{R}$ and Received May 29, 1987. 
$1 \leqq p<\infty$ (especially $H^{s}=H^{s}\left(\boldsymbol{R}^{n}\right)$ for $p=2$ ) be the Sobolev spaces which are the completion of $C_{0}^{\infty}\left(\boldsymbol{R}^{n}\right)$ with norms

$$
\|u\|_{s, p}=\left\|\mathscr{F}^{-1}\left(\left(1+|\xi|^{2}\right)^{s / 2} \hat{u}(\xi)\right)\right\|_{p} .
$$

Here ${ }^{\wedge}$ denotes the Fourier transformation and $\mathscr{F}^{-1}$ is its inverse. For any interval $I \subset \boldsymbol{R}$ and any Banach space $B$, we denote by $C^{k}(I ; B)$ the space of $B$ valued $C^{k}$-functions over $I$, and by $C_{w}(I ; B)$ the space of weakly continuous functions from $I$ to $B$, and by $C_{L}(I ; B)$ the space of functions from $I$ to $B$ that are strongly Lipschitz continuous. We denote by $C^{k}\left(I ; \mathscr{D}^{\prime}\right)$ the space of $\mathscr{D}^{\prime}$-valued functions $u(t)$ such that $\langle u(t), v\rangle$ is in $C^{k}(I)$ for any $v \in \mathscr{D}$.

We shall use the operator $\zeta(H)$ for suitable functions $\zeta(\cdot)$ as follows:

$$
\zeta(H) u=\mathscr{T}^{-1}(\zeta(\langle\xi\rangle) \hat{u}(\xi)) \quad \text { in } \mathcal{S}^{\prime} .
$$

where $\langle\xi\rangle=\left(1+|\xi|^{2}\right)^{1 / 2}$ and $\mathcal{S}^{\prime}$ means the tempered distribution.

Now we are ready to state our results.

THEOREM 1. Let $0<\gamma<n(n \geqq 1)$. Assume that $(\phi, \phi) \in H^{1} \cap L_{4 n /(2 n-r)} \times L_{2}$. Then there exists a weak solution $w(t)$ of (1.1) which satisfies the following:

$$
\begin{aligned}
& w(t) \in L_{\infty}\left(\boldsymbol{R} ; H^{1}\right) \cap C_{w}\left(\boldsymbol{R} ; H^{1}\right) \cap C_{L}\left(\boldsymbol{R} ; L_{2}\right) \cap C^{2}\left(\boldsymbol{R} ; \mathscr{D}^{\prime}\right), \\
& F(w(t)) \in L_{\infty}\left(\boldsymbol{R} ; L_{2 n /(n+\gamma)}\right) \cap C\left(\boldsymbol{R} ; \mathscr{D}^{\prime}\right) \\
& (w(t), v)=(\phi, \cos \{H t\} v)+\left(\psi, H^{-1} \sin \{H t\} v\right) \\
& \quad-\int_{0}^{t}\left(F(w(\tau)), H^{-1} \sin \{H(t-\tau)\} v\right) d \tau, \\
& \left\{\begin{array}{c}
\frac{d^{2}}{d t^{2}}(w(t), v)+(w(t),(-\Delta+1) v)+(F(w(t), v)=0 \\
(w(0), v)=(\phi, v), \frac{d}{d t}(w(0), v)=(\psi, v) .
\end{array}\right.
\end{aligned}
$$

Here $v \in C_{0}^{\infty}\left(\boldsymbol{R}^{n}\right)$ and (, ) is $L_{2}$-inner product. And we have the energy inequality

$$
E\left(w(t), \partial_{t} w(t)\right) \leqq E(\phi, \phi) \quad \text { for } \quad t \in \boldsymbol{R} .
$$

where

$$
E(\phi, \phi)=\frac{1}{2}\|\psi\|_{2}^{2}+\frac{1}{2}\|\phi\|_{1,2}^{2}+\frac{1}{4} V_{(n+\gamma) / 2} * f(\phi) \|_{2}^{2} .
$$

Theorem 2. Let $0<\gamma<\operatorname{Min}\{4, n\}(n \geqq 1)$ and $(\phi, \psi) \in H^{1} \times L_{2}$. Let $I$ be an open interval in $\boldsymbol{R}$ and $0 \in I$. Then there exists at most one $w(t)$ which satisfies (1.5) and

$$
w(t) \in L_{\infty}^{100}\left(I ; H^{1}\right) \quad \text { for } \quad 0<\gamma \leqq 3,
$$




$$
w(t) \in L_{\infty}^{\text {loc }}\left(I ; H^{1}\right) \cap L_{r}^{10 c}\left(I ; L_{p^{\prime}}\right) \quad \text { for } 3<\gamma<4,
$$

wher $1 / p^{\prime}=1 / 2-(\gamma-1) / 2 n$ and $1 / r=(\gamma-3) / 2$.

THEOREM 3. Let $0<\gamma<\operatorname{Min}\{4, n\} \quad(n \geqq 1)$.

(i) Let $(\phi, \phi) \in H^{1} \times L_{2}$. Then $w(t)$ which is obtained by Theorem 1 is unique and satisfies the following:

$$
\begin{aligned}
& w(t) \in C\left(\boldsymbol{R} ; H^{1}\right) \cap C^{1}\left(\boldsymbol{R} ; L_{2}\right) \quad \text { for } 0<\gamma \leqq 3, \\
& w(t) \in C\left(\boldsymbol{R} ; H^{1}\right) \cap C^{1}\left(\boldsymbol{R} ; L_{2}\right) \cap L_{r}^{\text {loc }}\left(\boldsymbol{R} ; L_{p^{\prime}}\right) \quad \text { for } 3<\gamma<4, \\
& E\left(w(t), \partial_{t} w(t)\right)=E(\phi, \phi) \quad \text { for } t \in \boldsymbol{R},
\end{aligned}
$$

where $r$ and $p^{\prime}$ are given in Theorem 2.

(ii) Let $(\phi, \psi) \in H^{k} \times H^{k-1}(k \in N$ (natural number) and $k \geqq 2)$. Then (1.1) has a unique solution $w(t)$ which satisfies

$$
w(t) \in \bigcap_{i=0}^{k} C^{i}\left(\boldsymbol{R} ; H^{k-i}\right) .
$$

Corollary. (i) If $k>n / 2+2, w(t)$ is in $C^{2}\left(\boldsymbol{R}^{n} \times \boldsymbol{R}\right)$.

(ii) If $k=\infty, w(t)$ is in $C^{\infty}\left(\boldsymbol{R}^{n} \times \boldsymbol{R}\right)$.

REMARK. (i) If $1<\gamma<\operatorname{Min}\{4, n\}$, we have $H^{1} \hookrightarrow L_{\mathbf{4} n /(2 n-\gamma)}$ by the Sobolev embedding theorem. So the initial condition $\phi \in H^{1} \cap L_{4 n /(2 n-\gamma)}$ becomes $\phi \in H^{1}$ in Theorem 2 and 3.

(ii) The upper bound $\operatorname{Min}\{4, n\}$ of $\gamma$ has been already appeared in the case of the nonlinear Schrödinger equation. (See [4] and [7].)

Theorem 1 is proved by the compactness method which were used by Segal in [12]. He used this method for the nonlinear Klein-Gordon equation with the power nonlinearity. (See also Reed [11] 5.) We can choose a convergent subsequence from solutions of the equation which approximate (1.1) by the double convolution mollifier due to Ginibre and Velo [3].

In the case $0<\gamma \leqq 3$ the same results of Theorem 2 and 3 have been already proved by [9]. Thus, we shall prove Theorem 2 and 3 in the case $3<\gamma<4$.

Theorem 2 is proved by the contraction method.

In order to prove Theorem 3, we show that a weak solution obtained by Theorem 1 becomes a regular solution. For this purpose we estimate the solutions of the approximating equation used for the proof of Theorem 1. This method has been already used by Ginibre and Velo [5] and Motai [10] in the case where $F(w)$ is the power nonlinearity. 


\section{Proof of Theorem 1 .}

First we approximate the nonlinear term by the double convolution mollifier due to Ginibre and Velo [3]. We choose an even non-negative function $h \in$ $C_{0}^{\infty}\left(\boldsymbol{R}^{n}\right)$ such that $\|h\|_{1}=1$. For any $j \in \boldsymbol{N}$ (natural number) we put

$$
F_{j}(u)=h_{j} *\left\{V_{r} * f\left(h_{j} * u\right) h_{j} * u\right\},
$$

where $h_{j}(x)=j^{n} h(j x)$. Coresponding to (2.1), we consider the Cauchy problem;

$$
\left\{\begin{array}{l}
\partial_{t}^{2} w_{j}(t)-\Delta w_{j}(t)+w_{j}(t)+F_{j}\left(w_{j}(t)\right)=0 \\
w_{j}(0)=h_{j} * \phi, \quad \partial_{t} w_{j}(0)=h_{j} * \psi
\end{array}\right.
$$

LEMmA 2.1. Let $0<\gamma<n \quad(n \geqq 1)$. Assume that $(\phi, \phi) \in H^{1} \cap L_{4 n /(2 n-r)} \times L_{2}$. Then for all $j \in \boldsymbol{N}(2.2)$ has a unique solution $w_{j}(t)$ such that

$$
w_{j}(t) \in \bigcap_{i=0}^{k} C^{i}\left(\boldsymbol{R} ; H^{k-i}\right) \quad \text { for any } \quad k \in \boldsymbol{N} .
$$

And $w_{j}(t)$ satisfies the integral equation in $H^{k}$;

$$
w_{j}(t)=w_{j}^{0}(t)-\int_{0}^{t} H^{-1} \sin \{H(t-\tau)\} F_{j}\left(w_{j}(\tau)\right) d \tau,
$$

where

$$
w_{j}^{0}(t)=\cos \{H t\} h_{j} * \phi+H^{-1} \sin \{H t\} h_{j} * \psi .
$$

In addition the conservation of energy holds;

$$
E_{j}\left(w_{j}(t), \partial_{t} w_{j}(t)\right)=E_{j}\left(h_{j} * \phi, h_{j} * \psi\right) \quad \text { for } \quad t \in \boldsymbol{R},
$$

where

$$
E_{j}(\phi, \phi)=\frac{1}{2}\|\phi\|_{2}^{2}+\frac{1}{2}\|\phi\|_{1,2}^{2}+\frac{1}{4}\left\|V_{(n+r) / 2} * f\left(h_{j} * \phi\right)\right\|_{2}^{2} .
$$

Proof. Applying Reed [11] Theorem 2 in section 1 to (2.2), we can show the existence of a unique global solution. Employing the same arguments as in Ginibre and Velo [3] Proposition 3.3, we can also prove (2.6).

We obtain the following lemma by the compactness method.

LEMma2.2. Let $w_{j}(t)(j \in N)$ be a solution of (2.2) obtained by Lemma 2.1. Then $\left\{w_{j}(t)\right\}$ has a convergent subsequence (again denoted by $\left\{w_{j}(t)\right\}$ ) as follows: For any compact interval $I \subset \boldsymbol{R}$ and any comsact subset $K \subset \boldsymbol{R}^{n}$

$$
w_{j}(t) \longrightarrow w(t) \quad \text { in } C\left(I ; L_{2}(K)\right) \quad \text { as } j \rightarrow \infty .
$$

Here $w(t)$ satisfies 


$$
w(t) \in L_{\infty}\left(\boldsymbol{R} ; H^{1}\right) \cap C_{w}\left(\boldsymbol{R} ; H^{1}\right) \cap C_{L}\left(\boldsymbol{R} ; L_{2}\right)
$$

Proof. Noting (2.6), the Ascoli-Arzela theorem yields (2.8) and (2.9). For details please refer to Segal [12] and Reed [11] 5 .

The following lemma is the well-known Sobolev's inequality.

LEMMA 2.3. Let $1<q<p<\infty$ and $0<\gamma<n(n \geqq 1)$. Then we have

$$
\left\|V_{\gamma} * u\right\|_{p} \leqq C\|u\|_{q}
$$

provided that

$$
\frac{1}{p}=\frac{1}{q}+\frac{\gamma}{n}-1
$$

PROOF. See Hörmander [8] Theorem 4.5.3 for a proof.

LEMMA 2.4. Let $0<\gamma<n(n \geqq 1)$. We have

$$
\begin{aligned}
\left|\int V_{\gamma} * f(w)(x) u(x) v(x) d x\right| & \leqq C\left\|V_{(n+\gamma) / 2} * f(w)\right\|_{2}\|u v\|_{2 n /(2 n-\gamma)} \\
& \leqq C\left\|V_{(n+\gamma) / 2} * f(w)\right\|_{2}\|u\|_{2}\|v\|_{2 n /(n-\gamma)}
\end{aligned}
$$

for suitable functions $u, v$ and $w$.

PROOF. Using the Plancherel theorem and the Schwartz inequality we have

$$
\begin{aligned}
& \int V_{\gamma^{*}} f(w)(x) u(x) v(x) d x=(2 n)^{-n} \int|\xi|^{(\gamma-n) / 2} \hat{f(w)}(\xi)|\xi|^{(\gamma-n) / 2} u \hat{v(\xi) d \xi} \\
& \leqq\left\|V_{(n+\gamma) / 2} * f(w)\right\|_{2}\left\|V_{(n+\gamma) / 2} *(u v)\right\|_{2} .
\end{aligned}
$$

It follows from Lemma 2, 3 and the Hölder inequality that

$$
\left\|V_{(n+\gamma) / 2} *(u v)\right\|_{2} \leqq C\|u v\|_{2 n /(2 n-\gamma)} \leqq C\|u\|_{2}\|v\|_{2 n /(n-\gamma)} .
$$

(2.13) and (2.14) show that (2.12) holds.

LEMMA 2.5. Let $0<\gamma<n(n \geqq 1)$. Let $w_{j}(t)$ be a solution of (2.2) obtained by Lemma 2.1. Then the following estimates holds:

$$
\begin{aligned}
& \left\|V_{(n+\gamma) / 2} * f\left(h_{j} * w_{j}(t)\right)\right\|_{2} \leqq C(\phi, \phi), \\
& \left\|V_{\gamma} * f\left(h_{j} * w_{j}(t)\right)\right\|_{2 n / \gamma} \leqq C(\phi, \phi), \\
& \left\|F_{j}\left(w_{j}(t)\right)\right\|_{2 n /(n+\gamma)} \leqq C(\phi, \phi)
\end{aligned}
$$

for $j \in \boldsymbol{N}$ and $t \in \boldsymbol{R}$, where $\boldsymbol{C}(\boldsymbol{\phi}, \phi)$ is a positive constant which is dependent on $(\phi, \phi)$ but independent of $t$ and $j$. 
Proof. Noting (2.6), we have (2.15) by Lemma 2.3.

From Lemma 2.4 it follows that

$$
\left|\int V_{\gamma} * f\left(h_{j} * w_{j}(t)\right) v(x) d x\right| \leqq C\left\|V_{(n+\gamma) / 2} * f\left(h_{j} * w_{j}(t)\right)\right\|_{2}\|v\|_{2 n /(2 n-\gamma)}
$$

for $v \in C_{0}^{\infty}\left(\boldsymbol{R}^{n}\right)$. Therefore we obtain (2.16) by (2.15), the density and the duality. Noting $\left\|w_{j}(t)\right\|_{2} \leqq C(\phi, \phi)$, (2.17) follows from (2.16) and the Hölder inequality.

LEMMA 2.6. Let I be any compact interval in $\boldsymbol{R}$. Let $\left\{w_{j}(t)\right\}$ be a convergent subsequence obtained by Lemma 2.2. Then it has the following properties:

$$
V_{(n+\gamma) / 2} * f\left(h_{j} * w_{j}(t)\right) \longrightarrow V_{(n+\gamma) / 2} * f(w(t))
$$

weakly in $L_{2}$ and uniformly on $I$ and

$$
F_{j}\left(w_{j}(t)\right) \longrightarrow F(w(t))
$$

weakly in $L_{2 n /(n+\gamma)}$ for $t \in I$ as $j \rightarrow \infty$.

In order to prove this lemma, we prepare two lemmas.

LEMMA 2.7. For any compact interval $I \subset \boldsymbol{R}$ and any compact subset $K \subset \boldsymbol{R}^{n}$ we have

$$
h_{j} * w_{j}(t) \longrightarrow w(t) \quad \text { in } \quad C\left(I ; L_{2}(K)\right) \quad \text { as } j \rightarrow \infty
$$

Proof. Noting (2.8), we can prove (2.21) easily. So we may omit the proof.

LEMMA 2.8. Let $0<\gamma<n$. For any compact interval $I \subset \boldsymbol{R}$ we have

$$
V_{\gamma} * f\left(h_{j} * w_{j}(t)\right) \longrightarrow V_{r} * f(w(t)) \quad \text { in } \mathscr{D}^{\prime}
$$

uniformly on $I$ as $j \rightarrow \infty$.

Proof. Let $v \in C_{0}^{\infty}\left(\boldsymbol{R}^{n}\right)$ and supp $v \subset\{x ;|x| \leqq R\}$. By the Fubini theorem we have

$$
\begin{aligned}
\int V_{r} *\left\{f\left(h_{j} * w_{j}(t)\right)-f(w(t))\right\} v(x) d x & =\int\left\{f\left(h_{j} * w_{j}(t)\right)-f(w(t))\right\} V_{r} * v(x) d x \\
& =\int_{|x| \leqq R+m}+\int_{|x| \geq R+m} \\
& =I_{1}+I_{2} .
\end{aligned}
$$

Here $m$ is a suitable number which will be chosen later. If $|x| \geqq R+m$, we 
have $|x-y| \geqq m$ for $|y| \leqq R$. Noting this, we obtain

$$
\begin{aligned}
\left|I_{2}\right| & \leqq m^{-r} \int\left|f\left(h_{j} * w_{j}(t)\right)-f(w(t))\right| d x \int|v(y)| d y \\
& \leqq m^{-r}\left(\left\|h_{j} * w_{j}(t)\right\|_{2}^{2}+\|w(t)\|_{2}^{2}\right)\|v\|_{1} .
\end{aligned}
$$

Next we estimate $I_{1}$. We have

$$
\left|I_{1}\right| \leqq \int_{|x| \leqq R+m}\left\{\left|f\left(h_{j} * w_{j}(t)\right)-f(w(t))\right| \int_{|y| \leqq R}|x-y|^{-\gamma}|v(y)| d y\right\} d x .
$$

It follows from $n-1-\gamma>-1$ that

$$
\int_{|y| \leqq R}|x-y|^{-r}|v(y)| d y \leqq C(2 R+m)^{n-r}\|v\|_{\infty} .
$$

This implies that

$$
\left|I_{1}\right| \leqq C(2 R+m)^{n-r}\left(\left\|w_{j}(t)\right\|_{2}+\|w(t)\|_{2}\right)\|v\|_{\infty}\left\|h_{j} * w_{j}(t)-w(t)\right\|_{L_{2}(|x| \leqq R+m)} .
$$

Choosing $m$ sufficiently large, we have (2.22) by (2.6), (2.9), (2.24), (2.27) and Lemma 2.7.

We are ready to prove Lemma 2.6.

Proof OF LemMA 2.6. As $0<(n+\gamma) / 2<n$, we have (2.19) by (2.15) and Lemma 2.8.

By (2.17) we obtain (2.20) if we can show that

$$
F_{j}\left(w_{j}(t)\right) \longrightarrow F(w(t)) \quad \text { in } \mathscr{D}^{\prime} \text { for } t \in I
$$

as $j \rightarrow \infty$. For $v \in C_{0}^{\infty}\left(\boldsymbol{R}^{n}\right)$ we have

$$
\begin{aligned}
\left(F_{j}\left(w_{j}(t)\right)-F(w(t)), v\right)= & \left(V_{\gamma} * f\left(h_{j} * w_{j}(t)\right) h_{j} * w_{j}(t), h_{j} * v-v\right) \\
& +\left(F\left(h_{j} * w_{j}(t)\right)-F(w(t)), v\right) \\
= & I_{1}+I_{2} .
\end{aligned}
$$

Lemma 2.4, (2.15) and (2.6) imply that

$$
\begin{aligned}
\left|I_{1}\right| & \leqq C\left\|V_{(n+\gamma) / 2} * f\left(h_{j} * w_{j}(t)\right)\right\|_{2}\left\|w_{j}(t)\right\|_{2}\left\|h_{j} * v-v\right\|_{2 n /(n-\gamma)} \\
& \leqq C(\phi, \phi)\left\|h_{j} * v-v\right\|_{2 n /(n-\gamma)} .
\end{aligned}
$$

We put

$$
\begin{aligned}
I_{2}= & \left(V_{\gamma} * f\left(h_{j} * w_{j}(t)\right)\left\{h_{j} * w_{j}(t)-w(t)\right\}, v\right) \\
& +\left(V_{\gamma} *\left\{f\left(h_{j} * w_{j}(t)\right)-f(w(t))\right\} w(t), v\right) \\
= & I_{21}+I_{22} .
\end{aligned}
$$

Again by Lemma 2.4 and (2.15) we have 


$$
\left|I_{21}\right| \leqq C(\phi, \psi)\left\|h_{j} * w_{j}(t)-w(t)\right\|_{L_{2}(\operatorname{supp} v)}\|v\|_{2 n /(n-r)} .
$$

We can rewrite $I_{22}$ as follows:

$$
I_{22}=\left(V_{r} *\left\{f\left(h_{j} * w_{j}(t)\right)-f(w(t))\right\}, w(t) v\right) .
$$

On the other hand it follows from (2.16) and Lemma 2.8 that

$$
V_{\gamma} * f\left(h_{j} * w_{j}(t)\right) \longrightarrow V_{\gamma} * f(w(t))
$$

weakly in $L_{2 n / \gamma}$ and uniformly on $I$ as $j \rightarrow \infty$. By the Hölder inequality and (2.6) we have $w(t) v \in L_{2 n /(2 n-\gamma)}$. Noting this, (2.34) implies that $I_{22} \rightarrow 0$ as $j \rightarrow \infty$. So (2.30), (2.32) and Lemma 2.7 show that (2.28) holds.

Now we are in a position to prove Theorem 1.

ProOF OF THEOREM 1. Let $\left\{w_{j}(t)\right\}$ be a convergent subsquence obtained by Lemma 2.2. We multiply $v \in C_{0}^{\infty}\left(\boldsymbol{R}^{n}\right)$ by (2.4) and integrate on $\boldsymbol{R}^{n}$. Then we have

$$
\begin{aligned}
\left(w_{j}(t), v\right)= & \left(h_{j} * \phi, \cos \{H t\} v\right)+\left(h_{j} * \phi, H^{-1} \sin \{H t\} v\right) \\
& -\int_{0}^{t}\left(F_{j}\left(w_{j}(\tau)\right), H^{-1} \sin \{H(t-\tau)\} v\right) d \tau
\end{aligned}
$$

Using the Hausdroff-Young inequality, we can show that $H^{-1} \sin \{H(t-\tau)\} v \in$ $L_{2 n /(n-\gamma)}$. Thus it follows from (2.20) that

$$
\left(F_{j}\left(w_{j}(\tau)\right), H^{-1} \sin \{H(t-\tau)\} v\right) \longrightarrow\left(F(w(\tau)), H^{-1} \sin \{H(t-\tau)\} v\right)
$$

as $j \rightarrow \infty$. By the Hölder inequality, (2.17) and the Hausdroff-Young inequality we have

$$
\begin{aligned}
\left(F_{j}\left(w_{j}(t)\right), H^{-1} \sin \{H(t-\tau)\} v\right) & \leqq\left\|F_{j}\left(w_{j}(\tau)\right)\right\|_{2 n /(n+\gamma)}\left\|H^{-1} \sin \{H(t-\tau)\} v\right\|_{2 n /(n-\gamma)} \\
& \leqq C(\phi, \phi)\|\hat{v}\|_{2 n /(n+\gamma)} .
\end{aligned}
$$

(2.36) and (2.37) mean that we can use the Lebesgue dominated convergence theorem. Thus letting $j \rightarrow \infty$ in (2.35), we obtain (1.5).

Noting $\phi \in L_{4 n /(2 n-\gamma)}$, (2.6) and (2.19) imply (1.7).

Next we show that

$$
(w(t), v) \in C^{2}(\boldsymbol{R}) \quad \text { for any } \quad v \in C_{0}^{\infty}\left(\boldsymbol{R}^{n}\right) .
$$

From (1.5) it follows that $(w(t), v) \in C^{1}(\boldsymbol{R})$ and

$$
\begin{aligned}
\frac{d}{d t}(w(t), v)= & -\left(\phi, H^{-1} \sin \{H t\} v+(\phi, \cos \{H t\} v)\right. \\
& -\int_{0}^{t}(F(w(\tau)), \cos \{H(t-\tau)\} v) d \tau
\end{aligned}
$$


If we show that

$$
(F(w(t)), v) \in C(\boldsymbol{R}) .
$$

(2.38) can be proved. Let $t \in \boldsymbol{R}$ and be fixed. Put

$$
\begin{aligned}
J(\eta)= & (F(w(t+\eta))-F(w(t)), v) \\
= & \left(V_{\gamma^{*}}\{f(w(t+\eta))-f(w(t))\} w(t), v\right) \\
& +\left(V_{r^{*}} f(w(t+\eta))\{w(t+\eta)-w(t)\}, v\right) \\
= & J_{1}(\eta)+J_{2}(\eta) .
\end{aligned}
$$

By (2.12) we obtain

$$
\left|J_{2}(\eta)\right| \leqq C\left\|V_{(n+\gamma) / 2} * f(w(t+\eta))\right\|_{2}\|w(t+\eta)-w(t)\|_{2}\|v\|_{2 n /(n-\gamma)} .
$$

From (1.7) and (2.9) it follows that $\left|J_{2}(\eta)\right| \rightarrow 0$ as $\eta \rightarrow 0$. By [2.3) and (2.16) we can show that

$$
V_{\gamma} * f\left(h_{j} * w_{j}(t)\right) \in C_{w}\left(\boldsymbol{R} ; L_{2 n / \gamma}\right) .
$$

(2.34) and (2.43) imply that

$$
V_{\gamma} * f(w(t)) \in C_{w}\left(\boldsymbol{R} ; L_{2 n / \gamma}\right) .
$$

Noting $w(t) v \in L_{2 n /(2 n-\gamma)}$, by (2.44) we have $\left|J_{1}(\eta)\right| \rightarrow 0$ as $\eta \rightarrow 0$. Then (2.40) is proved. Noting (2.9), (2.17) and (2.20), (1.3) and (1.4) have already been proved. (1.5) implies (1.6). Thus the proof of Theorem 1 is completed.

\section{Proof of Theosem 2.}

We begin with the well known estimates for the elementary solution of the linear Klein-Gordon equation.

Proposition 3.1. Lht $1<p \leqq 2$ and $1 / p+1 / p^{\prime}=1$. Put $\delta\left(p^{\prime}\right)=1 / 2-1 / p^{\prime}$.

(i) Let $p^{\prime}, s^{\prime}$ and $s$ satisfy

$$
(n+1) \delta\left(p^{\prime}\right) \leqq 1+s-s^{\prime} .
$$

Then we have for $g \in C_{0}^{\infty}\left(\boldsymbol{R}^{n}\right)$

$$
\left\|H^{-1} \sin \{H t\} g\right\|_{s^{\prime}, p^{\prime}} \leqq C|t|^{1+s-s^{\prime}-2 n \delta\left(p^{\prime}\right)}\|g\|_{s, p} .
$$

(ii) Put $1 / r=s^{\prime}+n \delta\left(p^{\prime}\right)-1$. Let $p^{\prime}, r$ and $s^{\prime}$ satisfy

$$
0 \leqq \frac{1}{r}<\frac{1}{2} \quad \text { and } \quad s^{\prime} \leqq 1-\frac{(n+1)}{2} \delta\left(p^{\prime}\right) .
$$

Then we have for $g \in C_{0}^{\infty}\left(\boldsymbol{R}^{n}\right)$

$$
\left\|H^{-1} \sin \{H t\} g\right\|_{L_{r}\left(R ; H_{p^{\prime}}^{s^{\prime}}\right)} \leqq C\|g\|_{2} .
$$


Proof. (i) See Brenner [1] Appendix 2 for a proof.

(ii) See Ginibre and Velo [5] Lemma 3.1 for a proof.

The following lemma is useful to estimate the nonlinear term.

LEMMA 3.2. Let $p, a, b$ and $q$ satisfy

$$
\frac{1}{p}=\frac{1}{a}+\frac{1}{b}+\frac{1}{q}+\frac{\gamma}{n}-1 \text { and } 1-\frac{\gamma}{n}<\frac{1}{a}+\frac{1}{b}<1 \text {. }
$$

Then we have

$$
\|F(u)-F(v)\|_{p} \leqq C\left(\|u-v\|_{a}\|u+v\|_{b}\|u\|_{q}+\|v\|_{a}\|v\|_{b}\|u-v\|_{q}\right)
$$

for suitable functions $u$ and $v$.

Proof. By the Hölder inequality and Lemma 2.3 we have (3.6). (2.11) yields (3.5).

ProOF OF THEOREM 2. As mentioned in the introduction, we will prove in the case $3<\gamma<4(n \geqq 4)$. Let $I$ be an open interval and $J$ be any finite interval such that $0 \in J \subset I$. Let $I_{0}$ be an interval such that $0 \in I_{0} \subset J$. Put

$$
X\left(I_{0}\right)=L_{\infty}\left(I_{0} ; H^{1}\right) \cap L_{r}\left(I_{0} ; L_{p^{\prime}}\right) .
$$

The norm of $X\left(I_{0}\right)$ is given by

$$
\|u\|_{X\left(I_{0}\right)}=\operatorname{Max}\left\{\|u\|_{L_{\infty}\left(I_{0} ; H^{1}\right)},\|u\|_{L_{r}\left(I_{0} ; L_{p^{\prime}}\right)}\right\} .
$$

From Lemma 2.4, Lemma 2.3 and the embedding $H^{1} \subseteq L_{4 n /(2 n-\gamma)}$ it follows that

$$
\begin{aligned}
\left|\int F(w(t)) v(x) d x\right| & \leqq\|w(t)\|_{1,2}^{3}\|v\|_{1,2} \\
& \leqq\|w\|_{X(J)}^{3}\|v\|_{1,2} .
\end{aligned}
$$

This means that $F(w(t)) \in H^{-1}$ for $t \in J$. Thus by (1.4) we have

$$
w(t)=w^{0}(t)-\int_{0}^{t} H^{-1} \sin \{H(t-\tau)\} F(w(\tau)) d \tau
$$

in $L_{2}$ for $t \in J$.

Let $w_{1}(t)$ and $w_{2}(t)$ be two solutions which satisfy the assumptioms of Theorem 2. From (3.8) we obtain

$$
w_{1}(t)-w_{2}(t)=-\int_{0}^{t} H^{-1} \sin \{H(t-\tau)\}\left[F\left(w_{1}(\tau)\right)-F\left(w_{2}(\tau)\right)\right] d \tau .
$$

By Proposition 3.1 (i) we have

$$
\left\|w_{1}(t)-w_{2}(t)\right\|_{p^{\prime}} \leqq C\left|\int_{0}^{t}\right| t-\left.\tau\right|^{3-\gamma} \| F\left(w_{1}(\tau)\right)-F\left(w_{2}(\tau) \|_{1, p} d \tau \mid .\right.
$$


Lemma 3.2 and the Sobolev embedding theorem yield that

$$
\begin{aligned}
& \left\|F\left(w_{1}(\tau)\right)-F\left(w_{2}(\tau)\right)\right\|_{1, p} \\
& \quad \leqq \\
& \quad C\left(\left\|w_{1}(\tau)\right\|_{1,2}+\left\|w_{2}(\tau)\right\|_{1,2}\right)\left(\left\|w_{1}(\tau)\right\|_{p^{\prime}}+\left\|w_{2}(\tau)\right\|_{p^{\prime}}\right)\left\|w_{1}(\tau)-w_{2}(\tau)\right\|_{1,2} \\
& \quad+C\left(\left\|w_{1}(\tau)\right\|_{1,2}+\left\|w_{2}(\tau)\right\|_{1,2}\right)^{2}\left\|w_{1}(\tau)-w_{2}(\tau)\right\|_{p^{\prime}} .
\end{aligned}
$$

By (3.10) we have

$$
\begin{aligned}
\left\|w_{1}(t)-w_{2}(t)\right\|_{p^{\prime}} \leqq & C\left\|w_{1}-w_{2}\right\|_{X\left(I_{0}\right)}\left(\left\|w_{1}\right\|_{X(J)}+\left\|w_{2}\right\|_{X(J)}\right) \\
& \times\left|\int_{0}^{t}\right| t-\left.\tau\right|^{3-\gamma}\left(\left\|w_{1}(\tau)\right\|_{p^{\prime}}+\left\|w_{2}(\tau)\right\|_{p^{\prime}}\right) d \tau \mid \\
& +C\left(\left\|w_{1}\right\|_{X\left(I_{0}\right)}+\left\|w_{2}\right\|_{X\left(I_{0}\right)}\right)^{2} \\
& \times\left|\int_{0}^{t}\right| t-\left.\tau\right|^{3-\gamma}\left\|w_{1}(\tau)-w_{2}(\tau)\right\|_{p^{\prime}} d \tau \mid
\end{aligned}
$$

As $3-\gamma>-1$, from the Young inequality we obtain

$$
\left\|w_{1}(t)-w_{2}(t)\right\|_{L_{r}\left(I_{0} ; L_{p^{\prime}}\right)} \leqq C\left|I_{0}\right|^{4-\gamma}\left(\left\|w_{1}\right\|_{X(J)}+\left\|w_{2}\right\|_{X(J)}\right)^{2}\left\|w_{1}-w_{2}\right\|_{X\left(I_{0}\right)} .
$$

Employing the same arguments as we obtain (3.11), we have

$$
\begin{aligned}
& \left\|F\left(w_{1}(\tau)\right)-F\left(w_{2}(\tau)\right)\right\|_{2} \\
& \quad \leqq C\left(\left\|w_{1}(\tau)\right\|_{1,2}+\left\|w_{2}(\tau)\right\|_{1,2}\right)\left(\left\|w_{1}(\tau)\right\|_{p^{\prime}}+\left\|w_{2}(\tau)\right\|_{p^{\prime}}\right)\left\|w_{1}(\tau)-w_{2}(\tau)\right\|_{p^{\prime}} .
\end{aligned}
$$

Hence it follows that

$$
\begin{aligned}
& \left\|w_{1}(t)-w_{2}(t)\right\|_{1,2} \\
& \quad \leqq C\left(\left\|w_{1}\right\|_{X(J)}+\left\|w_{2}\right\|_{X(J)}\right)\left|\int_{0}^{t}\left(\left\|w_{1}(\tau)\right\|_{p^{\prime}}+\left\|w_{2}(\tau)\right\|_{p^{\prime}}\right)\left\|w_{1}(\tau)-w_{2}(\tau)\right\|_{p^{\prime}} d \tau\right| .
\end{aligned}
$$

Noting $r>2$, from the Hölder inequality we obtain

$$
\left\|w_{1}(t)-w_{2}(t)\right\|_{1,2} \leqq C\left|I_{0}\right|^{(r-2) / r}\left(\left\|w_{1}\right\|_{X(J)}+\left\|w_{2}\right\|_{X(J)}\right)^{2}\left\|w_{1}-w_{2}\right\|_{X\left(I_{0}\right)} .
$$

(3.13) and (3.16) show that

$$
\left\|w_{1}-w_{2}\right\|_{X\left(I_{0}\right)} \leqq C\left|I_{0}\right|^{4-\gamma}\left(\left\|w_{1}\right\|_{X(J)}+\left\|w_{2}\right\|_{X(J)}\right)^{2}\left\|w_{1}-w_{2}\right\|_{X\left(I_{0}\right)} .
$$

Taking $\left|I_{0}\right|$ sufficiently small in (3.17), we obtain a inequality which implies that $w_{1}=w_{2}$ on $I_{0}$. Iterating this process, we can show that $w_{1}=w_{2}$ on $J$. As $J$ arbitrary, Theorem 2 is proved.

\section{Proof of Theorem 3 .}

In this section we restrict our attention to $3<\gamma<4(n \geqq 4)$, too. In order to investigate the regularity of a weak solution, we estimate the solutions of the approximating equation. 
LemMA 4.1. Let $3<\gamma<4(n \geqq 4)$. Let $(\phi, \phi) \in H^{1} \times L_{2}$ and $w_{j}(t)(j \in \boldsymbol{N})$ be $a$ solution of (2.2) obtained by Lemma 2.1. Let $p^{\prime}$ and $r$ be given in Theorem 2 . Then for any compact interval $I \subset \boldsymbol{R}$ there exists a positive constant $C(\phi, \phi, I)$ which is dependent on $(\phi, \phi)$ and I but independent of $j$ such that

$$
\left\|w_{j}\right\|_{L_{r}\left(I ; L_{p^{\prime}}\right)} \leqq C(\phi, \phi, I) \quad \text { for } \quad j \in N \text {. }
$$

Proof. It is sufficient to prove $[4.1)$ in the case $I=[0, \alpha]$. In the same way as we obtain (3.12) we have

$$
\left\|w_{j}(t)\right\|_{p^{\prime}} \leqq\left\|w_{j}^{0}(t)\right\|_{p^{\prime}}+C(\phi, \phi) \int_{0}^{t}|t-\tau|^{3-\gamma}\left\|w_{j}(\tau)\right\|_{p^{\prime}} d \tau
$$

Here we have used (2.6). By Propositon 3.1 (ii) and the Young inequality we have

$$
\begin{aligned}
&\left\|w_{j}\right\|_{L_{r}\left(I ; L_{p^{\prime}}\right)} \leqq C\left(\|\phi\|_{1,2}+\|\phi\|_{2}\right)+C(\phi, \phi)\left\|\int_{0}^{t}|t-\tau|^{3-r}\right\| w_{j}(\tau)\left\|_{p^{\prime}} d \tau\right\|_{L_{r}(I)} \\
& \leqq C\left(\|\phi\|_{1,2}+\|\phi\|_{2}\right)+C(\phi, \phi) \alpha^{4-r}\left\|w_{j}\right\|_{L_{r}\left(I ; L_{p^{\prime}}\right)} .
\end{aligned}
$$

We can verify the condition (3.3) easily. Choosing $\alpha$ to satisfy $C(\phi, \phi) \alpha^{4-\gamma} \leqq 1 / 2$, we have

$$
\left\|w_{j}\right\|_{L_{r}\left(I ; L_{p^{\prime}}\right)} \leqq C(\phi, \phi, I) \quad \text { for } \quad j \in \boldsymbol{N} \text {. }
$$

Next we show that (4.1) holds for any number $\alpha \in[0, \infty)$. Let $M$ be the supremum of the number $\alpha \in[0, \infty)$ so that $(4.1)$ holds with $I=[0, \alpha]$. We have already showed that $M>0$. If $M=\infty$, the lemma is proved. We assume that $M<\infty$. Let $\alpha<M$ and $I_{1}=[0, \alpha]$. From the definition of $M$ it follows that

$$
\left\|w_{j}\right\|_{L_{r}\left(I_{1} ; L_{p^{\prime}}\right)} \leqq C\left(\phi, \phi, I_{1}\right) \quad \text { for } \quad j \in \boldsymbol{N} .
$$

Let $\alpha<\beta$ and $I_{2}=[\alpha, \beta]$. Employing the same arguments as we obtain (4.3), we have

$$
\begin{aligned}
\left\|w_{j}\right\|_{L_{r}\left(I_{2} ; L_{p^{\prime}}\right) \leqq} & C\left(\|\phi\|_{1,2}+\|\phi\|_{2}\right) \\
& +C(\phi, \phi)\left\|\int_{\alpha}^{t}|t-\tau|^{3-\gamma}\right\| w_{j}(\tau)\left\|_{p^{\prime}} d \tau\right\|_{L_{r}\left(I_{2}\right)} \\
& +C(\phi, \phi)\left\|\int_{0}^{\alpha}|t-\tau|^{3-\gamma}\right\| w_{j}(\tau)\left\|_{p^{\prime}} d \tau\right\|_{L_{r}\left(I_{2}\right)} \\
= & J_{1}+J_{2}+J_{3} .
\end{aligned}
$$

From the same arguments of a proof of the Young inequality we obtain

$$
\begin{aligned}
& J_{2} \leqq C(\phi, \phi)(\beta-\alpha)^{4-r}\left\|w_{j}\right\|_{L_{r}\left(I_{2} ; L_{p^{\prime}}\right)}, \\
& J_{3} \leqq C(\phi, \phi) \beta^{4-r}\left\|w_{j}\right\|_{L_{r}\left(I_{1} ; L_{p^{\prime}}\right) .}
\end{aligned}
$$


Choosing $\beta$ near $\alpha$ to satisfy $C(\phi, \psi)(\beta-\alpha)^{4-\gamma} \leqq 1 / 2$, by $(4.5) \sim(4.8)$ we have

$$
\left\|w_{j}\right\|_{L_{r}\left([0, \beta] ; L_{p^{\prime}}\right)} \leqq C(\phi, \phi, \beta) \quad \text { for } \quad j \in N \text {. }
$$

Since the distence between $\alpha$ and $\beta$ depends on $C(\phi, \phi)$ only, we can choose $\alpha$ near $M$ to satisfy $M-\alpha<\beta-\alpha$. Hence (4.9) contradicts the definition of $M$.

LEMMA 4.2. Let $3<\gamma<4(n \geqq 4)$. Let $(\phi, \phi) \in H^{2} \times H^{1}$ and $w_{j}(t)(j \in \boldsymbol{N})$ be a solution of (2.2) obtained by Lemma 2.1. Let $1 / q^{\prime}=1 / 2-1 / 2 n$. Then for any compact interval $I \subset \boldsymbol{R}$ there exists a positive constant $C(\phi, \phi, I)$ which is dependent on $(\phi, \psi)$ and I but independent of $j$ such that

$$
\left\|w_{j}\right\|_{L_{\infty}\left(I ; H_{q^{\prime}}^{1}\right) \leqq C(\phi, \phi, I)} \text { for } \quad j \in N .
$$

Proof. Let $I=[0, \alpha]$. From (2.4) and Proposition 3.1 (i) it follows that

$$
\left\|w_{j}(t)\right\|_{1, q^{\prime}} \leqq\left\|w_{j}^{0}(t)\right\|_{1, q^{\prime}}+\int_{0}^{t}\left\|F_{j}\left(w_{j}(\tau)\right)\right\|_{1, q} d \tau .
$$

We can verify (3.1) easily. Applying Lemma 3.2 to $\left\|F_{j}\left(w_{j}(\tau)\right)\right\|_{1, q}$, we have

$$
\left\|F_{j}\left(w_{j}(\tau)\right)\right\|_{1, q} \leqq C\left\|w_{j}(\tau)\right\|_{p^{\prime}}^{2}\left\|w_{j}(\tau)\right\|_{1, q^{\prime}},
$$

where $p^{\prime}$ is given by Lemma 4.1. As the embedding $H^{2} \hookrightarrow H_{q^{\prime}}^{1}$ holds, from (4.11) and (4.12) we obtain

$$
\left\|w_{j}(t)\right\|_{1, q^{\prime}} \leqq C\left(\|\boldsymbol{\phi}\|_{2,2}+\|\boldsymbol{\phi}\|_{1,2}\right)+C\left\|w_{j}\right\|_{L_{\infty}\left(I ; H_{q^{\prime}}\right.} \int_{0}^{t}\left\|w_{j}(\tau)\right\|_{p^{\prime}}^{2} d \tau .
$$

From the Hölder inequality and Lemma 4.1 it follows that

$$
\left\|w_{j}\right\|_{L_{\infty}\left(I ; H_{q}^{1}\right)} \leqq C\left(\|\phi\|_{2,2}+\|\phi\|_{1,2}\right)+C(\phi, \phi, I) \alpha^{(r-2) / r}\left\|w_{j}\right\|_{L_{\infty}\left(I ; H_{q^{\prime}}^{1}\right)} .
$$

Here choosing $\alpha$ sufficiently small, we have

$$
\left\|w_{j}\right\|_{L_{\infty}\left(I ; H_{q}^{\prime}\right)} \leqq C(\phi, \phi, I) .
$$

Employing the same arguments of the proof of Lemma 4.1, we can show that (4.10) holds for any $\alpha \in[0, \infty)$. So we may omit its proof.

LEMMA 4.3. Under the same assumptions of Lemma 4.2. we have

$$
\left\|w_{j}\right\|_{L_{\infty}\left(I ; H^{2}\right)} \leqq C(\phi, \phi, I) \quad \text { for } \quad j \in \boldsymbol{N}
$$

for any compact interval $I \subset \boldsymbol{R}$. Here $C(\phi, \phi, I)$ is a positive constant which is dependent on $(\phi, \phi)$ and $I$ but independent of $j$.

Proof. From (2.4) it follows that

$$
\left\|w_{j}(t)\right\|_{2,2} \leqq C\left(\|\phi\|_{2,2}+\|\phi\|_{1,2}\right)+\int_{0}^{t}\left\|F_{j}\left(w_{j}(\tau)\right)\right\|_{1,2} d \tau .
$$


Applying Lemma 3.2 to $\left\|F_{j}\left(w_{j}(\tau)\right)\right\|_{1,2}$, we obtain

$$
\left\|F_{j}\left(w_{j}(\tau)\right)\right\|_{1,2} \leqq C\left\|w_{j}(\tau)\right\|_{1, q^{\prime}}^{2}\left\|w_{j}(\tau)\right\|_{2,2},
$$

where $q^{\prime}$ is given by Lemma 4.2. To note Lemma 4.2, we have

$$
\left\|w_{j}(t)\right\|_{2,2} \leqq C\left(\|\phi\|_{2,2}+\|\phi\|_{1,2}\right)+C(\phi, \phi, I) \int_{0}^{t}\left\|w_{j}(\tau)\right\|_{2,2} d \tau .
$$

The Gronwall inequality implies (4.16).

Now we give the estimates of the weak solution.

LEMMA 4.4. Let $w(t)$ be a weak solution of (1.1) obtained by Theorem 1 . Let $3<\gamma<4(n \geqq 4)$ and $I$ be any compact interval in $\boldsymbol{R}$.

(i) Let $(\phi, \phi) \in H^{1} \times L_{2}$. Then we have

$$
\|w\|_{L_{r}\left(I ; L_{p^{\prime}}\right)} \leqq C(\phi, \phi, I),
$$

where $C(\phi, \phi, I)$ is a positive constant which is dependent on $(\phi, \phi)$ and $I$, provided that

$$
\frac{1}{p^{\prime}}=\frac{1}{2}-\frac{r-1}{2 n} \text { and } \frac{1}{r}=\frac{r-3}{2} .
$$

(ii) Let $(\phi, \phi) \in H^{2} \times H^{1}$. Then we have

$$
\|w\|_{L_{\infty}\left(I ; H^{2}\right)} \leqq C(\phi, \phi, I),
$$

where $C(\phi, \phi, I)$ is a positive constant which is dependent on $(\phi, \psi)$ and $I$.

Proof. By (4.1), (4.16) and Lemma 2.2 we can choose a covergent subsequence (again denoted by $w_{j}(t)$ ) so that

$$
\begin{array}{ll}
w_{j}(t) \longrightarrow w(t) & \text { weakly in } L_{r}\left(I ; L_{p^{\prime}}\right), \\
w_{j}(t) \longrightarrow w(t) & \text { weakly in } H^{2} \text { and uniformly on } I
\end{array}
$$

as $j \rightarrow \infty$. Thus we have (4.20) and (4.22).

We prepare three lemmas on the regularity of the integral equation.

Lemma 4.5. Assume that for $i=0$ or 1

$$
F(w(t)) \in L_{1}^{\text {loc }}\left(\boldsymbol{R} ; H^{i}\right) .
$$

Then we have

$$
\int_{0}^{t} H^{-1} \sin \{H(t-\tau)\} F(w(\tau)) d \tau \in C\left(\boldsymbol{R} ; H^{1+i}\right) \cap C^{1}\left(\boldsymbol{R} ; H^{i}\right) .
$$

Proof. See Motai [9] Lemma 4.2 for a proof. 
Lemma 4.6. Assume that for $k \in \boldsymbol{N}$

$$
w(t) \in \bigcap_{i=0}^{k} C^{i}\left(\boldsymbol{R} ; H^{k-i}\right) .
$$

Then we have

$$
F(w(t)) \in \bigcap_{i=0}^{k} C^{i}\left(\boldsymbol{R} ; H^{k-i}\right) \quad \text { for } \quad 0<\gamma<\operatorname{Min}\{2 k, n\} .
$$

Proof. If we use Lemma 3.2 and the Sobolev embedding theorem, we can prove (4.28) easily. So we may omit a proof.

LEMMA 4.7. Assume that for $k \in \mathbf{N}$

$$
F(w(t)) \in \bigcap_{i=0}^{k} C^{i}\left(\boldsymbol{R} ; H^{k-i}\right) .
$$

Then we have

$$
\int_{0}^{t} H^{-1} \sin \{H(t-\tau)\} F(w(\tau)) d \tau \in \bigcap_{i=0}^{k+1} C^{i}\left(\boldsymbol{R} ; H^{k+1-i}\right)
$$

Proof. This result is well-known. So we may omit the proof.

We are in a positon to prove Theorem 3.

Proof OF THEOREM 3. (i) Let $w(t)$ be a weak solution obtained by Theorem 1. Since $w(t) \in L_{\infty}\left(\boldsymbol{R} ; H^{1}\right)$, from the same argument as we obtain (3.8) it follows that

$$
w(t)=w^{0}(t)-\int_{0}^{t} H^{-1} \sin \{H(t-\tau)\} F(w(\tau)) d \tau \quad \text { in } L_{2}
$$

for $t \in \boldsymbol{R}$. By $(\phi, \phi) \in H^{1} \times L_{2}$ we have

$$
w^{0}(t) \in C\left(\boldsymbol{R} ; H^{1}\right) \cap C^{1}\left(\boldsymbol{R} ; L_{2}\right) .
$$

Noting (3.14), from (1.7) we obtain

$$
\|F(w(t))\|_{2} \leqq C(\phi, \phi)\|w(t)\|_{p^{\prime}}^{2} .
$$

As $r>2$, Lemma 4.4 (i) and (4.32) imply (4.25). Hence by Lemma 4.5 we have (1.12).

The uniqueness of $w(t)$ follows from (1.12) and Theorem 2.

If we resolve (1.1) at initial time $t_{0} \in \boldsymbol{R}$ with a initial data $\left(w\left(t_{0}\right), \partial_{t} w\left(t_{0}\right)\right)$, by Theorem 1 we obtain

$$
E\left(w(t), \partial_{t} w(t)\right) \leqq E\left(w\left(t_{0}\right), \partial_{t} w\left(t_{0}\right)\right) \quad \text { for } \quad t \in \boldsymbol{R} .
$$

The uniqueness, (1.7) and (4.34) imply (1.13). 
(ii) We first note that for $(\phi, \phi) \in H^{k} \times H^{k-1}(k \geqq 2)$ we have

$$
w_{0}(t) \in \bigcap_{i=0}^{k} C^{i}\left(\boldsymbol{R} ; H^{k-i}\right) .
$$

In the case $k=2$ we have

$$
F(w(t)) \leqq C\|w(t)\|_{2,2}^{3}
$$

by Lemma 3.2 and the Sobolev embedding theorem. From Lemma 4.4 (ii) and Lemma 4.5 it follows that

$$
w(t) \in C\left(\boldsymbol{R} ; H^{2}\right) \cap C^{1}\left(\boldsymbol{R} ; H^{1}\right) .
$$

This implies that

$$
F(w(t)) \in C\left(\boldsymbol{R} ; H^{1}\right) \cap C^{1}\left(\boldsymbol{R} ; L_{2}\right) .
$$

By Lemma 4.7 we have

$$
w(t) \in \bigcap_{i=0}^{2} C^{i}\left(\boldsymbol{R} ; H^{2-i}\right) .
$$

In the case $k>2$ we can first obtain (4.39). Lemma 4.6 shows that

$$
F(w(t)) \in \bigcap_{i=0}^{2} C^{i}\left(\boldsymbol{R} ; H^{2-i}\right) .
$$

And Lemma 4.7 implies that

$$
w(t) \in \bigcap_{i=0}^{3} C^{i}\left(\boldsymbol{R} ; H^{3-i}\right) .
$$

Iterating this process, we can prove (1.14).

Corollary follows from the Sobolev lemma.

The proof Theorem 3 is completed.

\section{References}

[1] Brenner, P., On scattering and everywhere defined scattering operator for nonlinear Klein-Gordon equations, J. Differential equations, 56 (1985), 310-344.

[2] Chadam, J.M. and Glassey, R.T., Global existence of solutions to the Cauchy problem for time-dependent Hartree equations, J. Math. Phys., 16 (1975), 11221130.

[3] Ginibre, J. and Velo, G., On a class of nonlinear Schrödinger equation I, J. Funct. Analysis, 32 (1979), 1-32.

[4] - On a class of nonlinear Schrödinger equations with non local interaction, Math. Z., 170 (1980), 109-136.

[5] - The global Cauchy problem for the non linear Klein-Gordon equation, Math. Z., 189 (1985), 87-121.

[6] Glassey, R.T., Asymptotic behavior of solutions to a certain nonlinear SchrödingerHartree equation, Comm. Math. Phys., 53 (1977), 9-18.

[7] Hayashi, N. and Tsutsumi, Y., Scattering theory for Hartree type equations, Ann. 
Henri Poincare, Phys. Theor., 46 (1987), 187-213.

[8] Hörmander, L., The analysis of linear partial differential operators I, BerlinHeidelberg-New York, Springer 1983.

[9] Menzala, C.P. and Strauss, W.A., On a wave equation with a cubic convolution, J. Differential equations, 43 (1982), 93-105.

[10] Motai, T., Existence of global strong solution for nonlinear Klein-Gordon equation, to appear in Funkcialoj Ekvacioj.

[11] Reed, M., Abstract nonlinear wave equation, Lecture notes in mathematics, 507 (1976), Berlin-Heidelberg-New York, Springer.

[12] Segal, I.E., The global Cauchy problem for a relativistic scalar field with power interaction, Bull. Soc. Math. Fr., 91 (1963), 129-135.

[13] Strauss, W.A., Nonlinear scattering theory at low energy: sequel, J. Funct. Analysis, 43 (1981), 281-293.

Japanese Language School

The Tokyo University

of Foreign Studies

5-10-1, Sumiyoshi-cho,

Fuchu-shi, Tokyo, 183 Japan 